\title{
C-reactive protein and white matter microstructural changes in COVID-19 patients with encephalopathy
}

\author{
Alexandra Rhally ${ }^{1}$ - Alessandra Griffa ${ }^{2,3}$ (D) Stéphane $\mathrm{Kremer}^{4,5} \cdot$ Marjolaine Uginet $^{2} \cdot$ Gautier Breville $^{2}$. \\ Patrick Stancu $^{2} \cdot$ Frédéric Assal $^{2} \cdot$ Patrice H. Lalive ${ }^{2,6,7} \cdot$ Karl-Olof Lövblad $^{8} \cdot$ Gilles Allali $^{2,9}$
}

Received: 22 July 2021 / Accepted: 22 September 2021 / Published online: 28 October 2021

(c) The Author(s) 2021

\begin{abstract}
Encephalopathy is a neurological complication of COVID-19. The objective of this exploratory study is to investigate the link between systemic inflammation and brain microstructural changes (measured by diffusion-weighted imaging) in patients with COVID-19 encephalopathy. 20 patients with COVID-19 encephalopathy (age: $67.3 \pm 10.0$ years; 90\% men) hospitalized in the Geneva University Hospitals for a SARS-CoV-2 infection between March and May 2020 were included in this retrospective cohort study. COVID-19 encephalopathy was diagnosed following a comprehensive neurobiological evaluation, excluding common causes of delirium, such as hypoxemic or metabolic encephalopathy. We investigated the correlation between systemic inflammation (measured by systemic C-reactive protein (CRP)) and brain microstructural changes in radiologically normal white matter (measured by apparent diffusion coefficient (ADC)) in nine spatially widespread regions of the white matter previously associated with delirium. Systemic inflammation $(\mathrm{CRP}=60.8 \pm 50.0 \mathrm{mg} / \mathrm{L})$ was positively correlated with ADC values in the anterior corona radiata $(p=0.0089)$, genu of the corpus callosum $(p=0.0064)$ and external capsule $(p=0.0086)$ after adjusting for patients' age. No statistically significant association between CRP and ADC was found in the other six white matter regions. Our findings indicate high risk of white matter abnormalities in COVID-19 encephalopathy patients with high peripheral inflammatory markers, suggesting aggressive imaging monitoring may be warranted in these patients. Future studies should clarify a possible specificity of the spatial patterns of CRP-white matter microstructure association in COVID-19 encephalopathy patients and disentangle the role of individual cytokines on brain inflammatory mechanisms.
\end{abstract}

Keywords COVID-19 $\cdot$ DWI $\cdot$ CRP $\cdot$ Encephalopathy $\cdot$ Inflammation $\cdot$ White matter

Alessandra Griffa

alessandra.griffa@gmail.com

1 Faculty of Medicine, University of Geneva, Geneva, Switzerland

2 Department of Clinical Neurosciences, Division of Neurology, Geneva University Hospitals and Faculty of Medicine, University of Geneva, Geneva, Switzerland

3 Institute of Bioengineering, Center of Neuroprosthetics, Ecole Polytechnique Fédérale de Lausanne (EPFL), Campus Biotech-H4 3232.080 (H4 building), Chemin des Mines 9, Case postale 60, CH-1211 Geneva, Switzerland

4 Service d'imagerie 2, Hôpitaux Universitaires de Strasbourg, Strasbourg, France
5 Engineering Science, Computer Science and Imaging Laboratory (ICube), Integrative Multimodal Imaging in Healthcare, UMR 7357, University of Strasbourg-CNRS, Strasbourg, France

6 Diagnostic Department, Division of Laboratory Medicine, Geneva University Hospitals, Geneva, Switzerland

7 Department of Pathology and Immunology, Faculty of Medicine, University of Geneva, Geneva, Switzerland

8 Division of Neuroradiology, Geneva University Hospitals and University of Geneva, Geneva, Switzerland

9 Division of Cognitive and Motor Aging, Department of Neurology, Albert Einstein College of Medicine, Yeshiva University, Bronx, NY, USA 


\section{Introduction}

Coronavirus disease 2019 (COVID-19) induced by severe acute respiratory syndrome coronavirus-2 (SARS-CoV-2) infection has been associated with neurological complications, such as idiopathic encephalopathy (Helms et al. 2020a, b). The clinical spectrum of the COVID-19 encephalopathy is broad, ranging from delirium to coma. An inflammatory hypothesis has been suggested for the neuropathogenesis of this encephalopathy (Teuwen et al. 2020; Najjar et al. 2020; Fotuhi et al. 2020). Although a possible disruption of brain microstructural integrity has been demonstrated in COVID19 patients (Lu et al. 2020; Newcombe et al. 2020), the relationship between systemic inflammation and microstructural brain changes has been poorly investigated in patients with COVID-19 encephalopathy.

The apparent diffusion coefficient (ADC), a measure derived from diffusion-weighted imaging (DWI) quantifying the local amount of water molecule diffusion, has been largely used to assess white matter microstructure (Alexander et al. 2007). White matter changes in the corpus callosum, thalamocortical, limbic, and cerebellar circuits have been associated with delirium (Nitchingham et al. 2018). To investigate the link between systemic inflammation and brain microstructural changes in COVID-19 encephalopathy patients, we studied the association between C-reactive protein (CRP) levels and ADC in nine white matter regions previously associated with delirium.

\section{Materials and methods}

\section{Standard protocol approvals, registrations, and patients consents}

The study was approved by the institutional review board of Geneva University Hospitals (protocol \#2020-01206approved May 25, 2020) and has been performed in accordance with the ethical standards laid down in the 1964 Declaration of Helsinki and its later amendments.

De-identified data will be made available to qualified investigators upon written request to the last author.

\section{Patient cohort}

From March to May 2020, 41 patients had been diagnosed with COVID-19 encephalopathy out of 707 COVID19 patients hospitalized in Geneva University Hospitals. SARS-CoV-2 infection was confirmed by a positive SARSCoV-2 reverse transcription polymerase chain reaction (RT-PCR) assay from a nasopharyngeal swab at the time of hospitalization. Diagnosis of COVID-19 encephalopathy was defined by a rapidly developing (less than 4 weeks) brain pathological process leading to delirium, decreased level of consciousness or coma (Helms et al. 2020b), excluding classical etiologies for delirium such as hypoxemia, electrolyte disturbances, infection, drug or alcohol toxicity, metabolic disorders, low perfusion state or acute central nervous system conditions such as meningitis. The severity of the encephalopathy was assessed with the Richmond Agitation-Sedation Scale (RASS) (a patient with an RASS score of -5 being unarousable (unresponsive to voice and physical stimulation) and a patient with an RASS score of +4 being combative (attempts to stand or get out of bed; danger to self or staff)), and with the duration of encephalopathy. Clinical data, CSF RT-PCR for SARS-CoV-2, CSF white blood cell count (WBC), CSF/serum quotient of albumin (QAlb), and blood CRP levels at the time of brain magnetic resonance imaging (MRI) were retrospectively collected from the patients' records. Patients with incomplete MRI data, image processing failure or more than 48-h delay between CRP assessment and MRI were excluded. Twenty patients were included in the final analyses (67.3 \pm 10.0 years; 18 men), of which 11 had CSF analysis (Table1).

\section{Brain imaging}

All 20 patients underwent an MRI scan at Geneva University Hospitals on a 1.5 Tesla Philips Ingenia system equipped with a multi-channel head coil. The MRI scan included a structural and a diffusion-weighted sequence with the following parameters: 2D gadolinium-enhanced T1-weighted gradient echo sequence, transverse acquisition, in-plane resolution $0.4 \times 0.4 \mathrm{~mm}^{2}$, acquisition matrix $=512 \times 512$ pixels; slice thickness $=5 \mathrm{~mm}$, spacing between slices $=5.3 \mathrm{~mm}$, number of slices $=30$, number of averages $=2$, flip angle $=80^{\circ}, \mathrm{TE}=2.4 \mathrm{~ms}$, TR $=259 \mathrm{~ms}$; 2D diffusion-weighted spin echo sequence, transverse acquisition, in-plane resolution $=1.3 \times 1.3 \mathrm{~mm}^{2}$, acquisition matrix $=176 \times 176$ pixels, slice thickness $=4 \mathrm{~mm}$, spacing between slices $=4.4 \mathrm{~mm}$, number of slices $=34$, number of averages $=2$, flip angle $=90^{\circ}, \mathrm{TE}=75 \mathrm{~ms}, \mathrm{TR}=4061 \mathrm{~ms}$, diffusion $b$-value $=1000 \mathrm{~s} / \mathrm{mm}^{2}$.

\section{Image analyses}

The average DWI b1000 images were inspected by two certified neuroradiologists to assess the presence of stroke. Acute stroke lesions were semi-automatically segmented on the b1000 images using the Clusterize SPM12 toolbox (Clas et al. 2012). The T1-weighted volumes were segmented into brain tissue compartments and co-registered to the diffusion volumes using SPM12 (Penny et al. 2006). Regions of interest in the white matter were extracted using an automatic 
Table 1 Clinical characteristics

\begin{tabular}{|c|c|}
\hline & All participants \\
\hline No. of participants & 20 \\
\hline Age (years), mean $\pm \mathrm{SD}$ (range) & $67.3 \pm 10.0(46.6$ to 80.03$)$ \\
\hline Sex, men $N(\%)$ & $18(90 \%)$ \\
\hline $\mathrm{BMI}^{\mathrm{a}}\left(\mathrm{kg} / \mathrm{m}^{2}\right)$, mean $\pm \mathrm{SD}($ range $)$ & $27.3 \pm 2.8(21.9$ to 31.2$)$ \\
\hline Stroke, $N(\%)$ & $2(10 \%)$ \\
\hline $\mathrm{CRP}(\mathrm{mg} / \mathrm{L})$, mean $\pm \mathrm{SD}$ (range) & $60.8 \pm 50.0(8.0$ to 153.0$)$ \\
\hline \multicolumn{2}{|l|}{ Severity of encephalopathy } \\
\hline RASS $^{\mathrm{b}}$, median (range) & $-1(-4$ to -2$)$ \\
\hline COVID-19 encephalopathy duration (days), mean \pm SD (range) & $13.6 \pm 8.6(4.0$ to 32.0$)$ \\
\hline \multicolumn{2}{|l|}{ CSF analyses } \\
\hline White blood cell count, mean \pm SD (range) & $2.09 \pm 2.47(0.00$ to 9.00$)$ \\
\hline $\mathrm{CSF} /$ serum quotient of albumin, mean $\pm \mathrm{SD}$ (range) & $12.37 \pm 7.49(4.56$ to 28.58$)$ \\
\hline
\end{tabular}

segmentation based on the ICBM-DTI-81 white matter atlas (Mori et al. 2008). In particular, the atlas was warped to individual T1-weighted images to identify white matter regions in native space. Voxel not belonging to the white matter according to the SPM12 segmentation were excluded from the regions of interest (threshold on individual white matter tissue probability maps: 0.8 ). In addition, to ensure that results were not driven by the presence of stroke, all analyses were repeated excluding the patients with acute stroke $(N=2)$. Based on previous literature on delirium (Nitchingham et al. 2018), nine bilateral white matter regions were retained for further analyses, namely, the genu and splenium of the corpus callosum, the posterior thalamic radiation, the anterior corona radiata, the cingulum, the uncinate fasciculus, the hippocampal white matter, the external capsule and the middle cerebellar peduncle. Of note, the uncinate fasciculus is part of a dense network of white matter fibers that interconnect different structures of the olfactory sensory pathway, and the investigation of this region may further elucidate the possible implication of the olfactory route regarding neuro-invasion (Lopez-Elizalde et al. 2018). An average $\mathrm{ADC}$ value was computed for each white matter region (average between left and right hemispheres), excluding voxels belonging to acute stroke lesions. In addition, analyses were repeated considering regions belonging to the left and right hemispheres separately to assess possible lateralization effects. Image analyses were performed with MATLABv9.7 and SPM12.

\section{Statistical analyses}

Linear regression analyses were used to assess the associations between the average ADC in nine white matter regions and CRP levels. Age was added as covariate. False discovery rate was controlled at FDR $=0.05$ to correct for multiple comparisons (Benjamini and Hochberg 1995). Spearman's rank correlation was used in exploratory analyses to assess possible relationships between CRP levels or ADC in different brain regions, and severity of encephalopathy as quantified with the RASS score and the duration of encephalopathy.

\section{Results}

The clinical characteristics of the COVID-19 encephalopathy patients are presented in Table 1 . The majority of patients were men and their CRP levels ranged from 8.0 to $153.0 \mathrm{mg} / \mathrm{L}$. The RASS scores ranged from -4 to -2 , with a median of -1 , indicating that the majority of patients suffered a mild form of encephalopathy. Duration of encephalopathy ranged from 4 to 32 days, with a mean duration of $13.6 \pm 8.6$ days. 11 out of 20 patients had a CSF analysis: the mean CSF white blood cell count was $2.09 \pm 2.47$ leukocytes $/ \mathrm{mm}^{3}$, whereas CSF/serum quotient of albumin (QAlb-measured in 8 out of 20 patients) was increased in $75 \%$ (mean QAlb $=12.37 \pm 7.49)$. CSF RT-PCR for SARSCoV-2 was negative for all patients (measured in 10 out of 20 patients).

Regression analyses with the average ADC in a white matter region as predicted variable, the CRP level as predictor variable, and the age as covariate, showed that higher CRP levels were significantly associated with increased $\mathrm{ADC}$ in the genu of the corpus callosum $(\beta=0.60$, $t(13)=3.16, p=0.0064)$, anterior corona radiata $(\beta=0.60$, $t(15)=2.95, p=0.0089)$, and external capsule $(\beta=0.61$, $t(15)=2.97, p=0.0068)$ (Fig. 1). These associations survived multiple comparison correction. No CRP-ADC association was found in the other six white matter regions. Repeating the analyses only on patients with no acute 
(a)

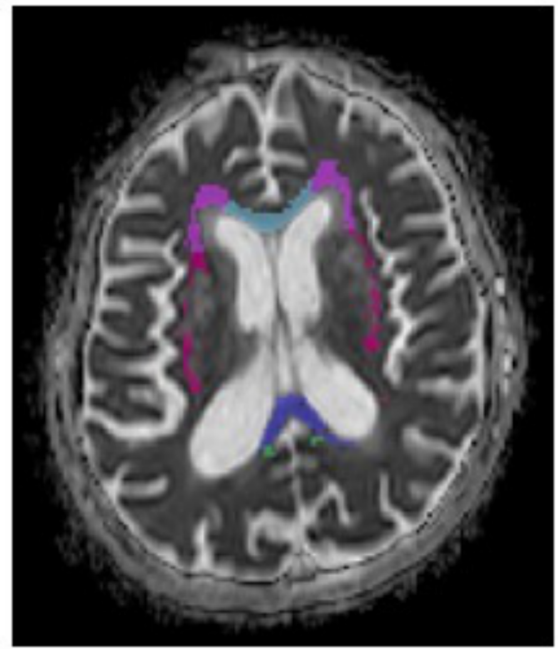

(c)

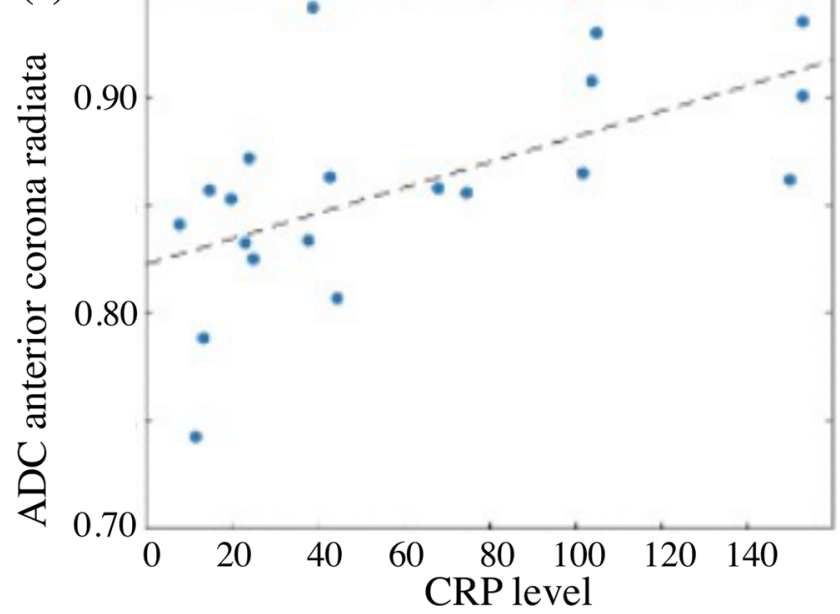

Fig. 1 Association between systemic inflammation and white matter microstructure. a Example of ADC map. Colored regions represent the genu of the corpus callosum (light blue), anterior corona radiata (lilac), external capsule (violet), splenium of the corpus callosum (dark blue) and cingulum (green). b-d Scatter plots representing the

stroke $(N=18)$ did not change results (genu of the corpus callosum: $\beta=0.49, t(11)=2.58, p=0.023$; anterior corona radiata: $\beta=0.69, t(13)=3.44, p=0.0036$; external capsule: $\beta=0.66, t(13)=3.11, p=0.0072$; associations between anterior corona radiata and external capsule ADC, and CRP levels survived FDR correction). No hemispheric lateralization of CRP-ADC associations was found when performing the analyses considering the left and right hemisphere separately (linear associations between CRP and ADC were significant with uncorrected $p$ value $<0.05$ for both left and right anterior corona radiata $(\beta=0.58, t(15)=2.86$, $p=0.011 ; \beta=0.60, t(15)=2.75, p=0.014$; respectively $)$, and left and right external capsule $(\beta=0.51, t(15)=2.32$, $p=0.033 ; \beta=0.62, t(15)=3.11, p=0.0063$; respectively) . Finally, there were no significant associations between CRP
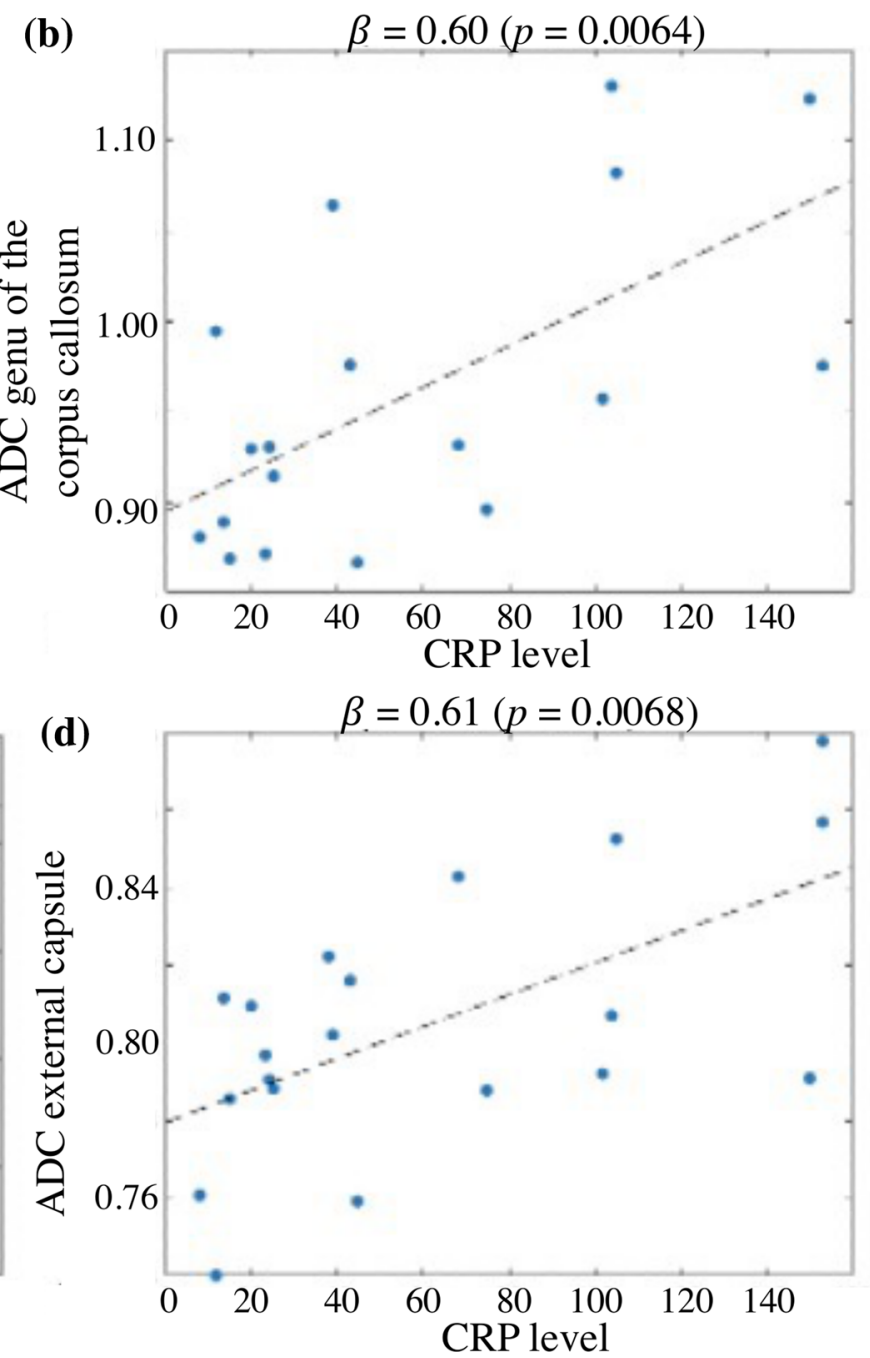

association between CRP levels and average ADC in the genu of the corpus callosum, anterior corona radiata and external capsule. Standardized beta coefficients and $p$ values are reported. Each dot in the plots represents a patient; the dashed lines represent the best linear fits

levels or ADC in different brain regions, and severity of encephalopathy as measured with the RASS score or the encephalopathy duration.

\section{Discussion}

In this preliminary study, we found that in COVID-19 encephalopathy patients, systemic inflammation (measured by CRP levels at the time of brain MRI) is associated with white matter microstructural changes (measured by ADC) in the anterior corona radiata, genu of the corpus callosum and external capsule. This association was specific to frontal (genu of the corpus callosum, anterior corona radiata) and cholinergic (external capsule) projections-no association 
was found in other limbic, thalamocortical, cerebellar, or olfactory regions. These findings are consistent with the cognitive profile of COVID-19 patients with encephalopathy, mainly presenting a fronto-subcortical syndrome (Beaud et al. 2020), and with the pathogenesis of delirium that can be triggered by acute inflammation (Wilson et al. 2020). Further analyses including comprehensive neuropsychological assessments are needed to understand whether neuropathological mechanisms related to systemic inflammation may impact frontal lobe functions.

The presence of an association between systemic inflammation and white matter microstructure in the corpus callosum is coherent with studies describing cytokine-induced injury in the corpus callosum in critical illnesses, such as acute respiratory distress syndrome and COVID-19 (Cannac et al. 2020), and a more frequent occurrence of white matter neurological events in this very same region in COVID-19 patients (Parsons et al. 2020). Furthermore, the increased quotient albumin reported in the majority of our patients with available CSF analyses suggests a blood-brain barrier disruption associated with this systematic inflammation, which may explain the observed white matter microstructural changes. However, fronto-cholinergic white matter microstructural changes may be particularly prevalent in COVID-19 encephalopathy, as research on other pathogendriven encephalopathies found different brain regions. For example, acute necrotizing encephalopathy has been associated with restricted diffusion imaging in the centrum semiovale, thalami, pontine tegmentum, cerebellar vermis, and deep cerebellar hemispheres (Bailey 2020). In the case of influenza-associated acute encephalopathy, restricted diffusion has been found in widespread white matter regions (Tsuchiya et al. 2000) and in the hippocampus (Koll et al. 2020). Moreover, the CRP-ADC associations in the anterior corona radiata and the external capsule highlighted by our analyses are consistent with previous research conducted on COVID-19 patients (Lu et al. 2020). However, we did not find any hemispheric lateralization of association between CRP and ADC changes in our analyses. Associations between CRP levels and white matter microstructure should be further investigated in future studies on larger cohorts, to explore the possible spatial specificity of effects to different virus-related encephalopathies.

White matter microstructure was assessed with the ADC, a measure of water molecule diffusion in brain tissues. ADC changes are considered to unspecifically reflect multiple microstructural mechanisms including axonal degeneration, loss of microstructural integrity as well as local inflammation (Alexander et al. 2007). Our findings suggest that systemic inflammation may support one or more of these neuropathological mechanisms resulting in ADC alteration. Inclusion of more advanced diffusion MRI sequences beyond DWI and diffusion tensor imaging remains mandatory for a thorough characterization of the microstructural mechanisms relating to inflammation, which, however, is impractical in critical clinical settings (Novikov et al. 2018; Alexander et al. 2017).

Our exploratory correlation analyses between systemic inflammation or white matter microstructure and the severity of encephalopathy, as recorded with the RASS score and the duration of encephalopathy, did not show any significant association between these variables. Various alternate mechanisms may contribute to the severity of encephalopathy, such as medication or comorbidities. Furthermore, delirium is highly fluctuating, and a RASS score recorded at a single time point may not fully represent the patients' clinical condition. The use of more sensitive measures of encephalopathy may help to better address the relationship between biological markers and clinical presentation.

Various mechanisms have been suggested to explain COVID-19 encephalopathy, involving a direct viral invasion through the olfactory retrograde route or an inflammatory mechanism during the so-called cytokine storm inducing blood-brain barrier (BBB) alterations (Iadecola et al. 2020). Several findings support the latter hypothesis, such as an increased CSF/serum quotient albumin in COVID19 encephalopathy patients suggestive of a leaky BBB, and SARS-CoV-2-associated cytokines (interleukin-6, IL-1 $\beta$, tumor necrosis factor and IL-17, among others) which are known to disrupt the BBB (Iadecola et al. 2020). Moreover, COVID-19 patients with encephalopathy have negative PCR SARS-CoV-2 in the CSF, suggesting that encephalopathy is not directly related to viral infection but to the inflammatory response. Post-mortem biopsy evidence of direct viral involvement in the brain or olfactory nerve is limited although this might be due to technical limitations (Mukerji and Solomon 2021; Butowt et al. 2021). On this line, we did not find any significant associations between systemic inflammation and white matter microstructure of the uncinate fasciculus, a fiber bundle that is part of the olfactory pathways (Lopez-Elizalde et al. 2018). The current findings showing an association between systemic inflammation and white matter microstructure in frontal and cholinergic projections support the inflammatory hypothesis.

Associations between serum lactate dehydrogenase (LDH) levels and DTI metrics have been observed in COVID-19 patients in a 3-month follow-up study (Lu et al. 2020), which is in line with our conclusion regarding the associations between microstructural changes in the white matter and CRP. Elevated LDH is a consequence of tissue damage (Augoff et al. 2015) and has been observed in various pathologies, such as encephalitis, ischemic stroke or head injuries (Valvona et al. 2016). Moreover, LDH levels have also been correlated with acute encephalopathy and could be related to prognosis as a significant difference in serum LDH levels was observed between pediatrics patients 
with and without developmental regression following acute encephalopathy (Motojima et al. 2016). Future research should evaluate if this association between serum LDH levels and DTI metrics in COVID-19 patients is related to neurological complications, such as encephalopathy, and if it is the case, whether it is a good predictor of outcome regarding neurological sequelae.

The association between systemic inflammation and white matter microstructure highlighted by our results is a snapshot of COVID-19 patients at the time of clinical presentation of encephalopathy. Regarding the long-term consequences of COVID-19 encephalopathy, recent studies suggest that this encephalopathy could have long-term effects on brain and cognition. First, microstructural changes in the white matter have been reported not only at the time of infection, but also up to 3 months after hospital discharge (Lu et al. 2020; Raman et al. 2021). A longitudinal PET study showed brain hypometabolism in prefrontal, insular, and middle temporal regions, with abnormalities persisting at 6 months follow-up (Kas et al. 2021). These findings indicate that brain integrity remains affected even months after SARS-CoV-2 infection. Persistent neuroinflammation has also been proposed as a possible explanation regarding long-term MRI changes seen in COVID-19 patients (Goldberg et al. 2020), and follow-up inflammatory markers correlate with severity of COVID-19 at admission (Raman et al. 2021). Second, impaired cognitive performances have been observed in COVID-19 patients compared to a control group in a follow-up study (Raman et al. 2021). Moreover, the incidence of neurological or psychiatric diagnoses-including dementia-following COVID-19 has been estimated at 34\%, higher than in a control group of patients who had influenza or other respiratory tract infection, and it increases to $62 \%$ for patients who presented a COVID-19 encephalopathy (Taquet et al. 2021). COVID-19, especially when complicated by COVID-19 encephalopathy, may therefore reveal, amplify, or accelerate subjacent neurological or psychiatric diagnosis and may lead to long-term clinical sequelae.

Although this highly selected sample of COVID-19 encephalopathy patients with brain imaging during acute presentation and CRP assessment at the time of imaging are major strengths of this exploratory study, the retrospective nature of this study has limitations. First, only blood C-reactive protein levels were available for these patients at the time of MRI, which limits our ability to draw conclusions using other inflammatory markers than CRP that might be more specific to central nervous system inflammation. Future studies should include a cytokine profile to disentangle the pathogenesis of this encephalopathy and other inflammatory markers, such as fibrinogen and IL-6, as CSF cytokine alterations have been highlighted in COVID-19 encephalopathy patients (Benameur et al. 2020). Second, we did not compare COVID-19 encephalopathy patients with another group of patients with virus-related encephalopathy (e.g., influenza), or with a group of Intensive Care Unit patients (independently from the type of pathology). Therefore, we cannot conclude that our results are specific to SARS-CoV-2 infection. Future studies should compare the strength of association between CRP and ADC values between different pathogen-driven encephalopathy groups to be able to conclude if these associations are specific to certain pathogens or to pathogen-related encephalopathies in general. Indeed, CRP levels have been correlated with white matter lesions in multiple pathologies not related to COVID-19, such as cerebral small vessel disease, neurodegenerative processes and obesity (Mitaki et al. 2015; Eagen et al. 2012; Lampe et al. 2018), which may occur as comorbidities in COVID-19 encephalopathy patients. The limited sample size of this preliminary study did not allow to control for confounders and comorbidities that could affect both the white matter integrity and the CRP values. We believe that a robust investigation in this direction will need to include larger samples and multiple patient groups to disentangle the role of specific pathophysiological mechanisms, inflammation markers and brain structure. In particular, it will be of interest to understand whether the fronto-cholinergic spatial patterns of association between white matter integrity and systemic inflammation are specific to COVID-19 encephalopathy. Finally, in this study white matter microstructure was assessed with the ADC parameter only. Future studies including more advanced diffusion MRI sequences as well as T2/FLAIR and susceptibility weighted imaging MRI contrasts among others (O'Donovan et al. 2021; Rahmanzade et al. 2020) are needed for a thorough characterization of white matter signal abnormalities and cerebrovascular factors to further explore the inflammatory damage and its relation to viral infections.

\section{Conclusions}

In conclusion, our findings demonstrate an association between a peripheral inflammatory marker and white matter microstructural changes in frontal and cholinergic projections, supporting an inflammatory pathogenesis to COVID19 encephalopathy. These results may provide a rationale to support the use of anti-inflammatory drugs for treating this encephalopathy.

Funding Open Access funding provided by Université de Genève. This study was funded by a donor from the Private Foundation of the Geneva University Hospitals.

Availability of data and material De-identified data will be made available to qualified investigators upon written request to the last author. 
Code availability Not applicable.

\section{Declarations}

Conflict of interest All authors declare no conflict of interest.

Ethics approval This study was approved by the institutional review board of Geneva University Hospitals (protocol \#2020-01206approved May 25, 2020) and has been performed in accordance with the ethical standards laid down in the 1964 Declaration of Helsinki and its later amendments.

Consent to participate Patients gave standard consent to Geneva University Hospitals, which approved the usage of human subjects for this study.

\section{Consent for publication Not applicable.}

Open Access This article is licensed under a Creative Commons Attribution 4.0 International License, which permits use, sharing, adaptation, distribution and reproduction in any medium or format, as long as you give appropriate credit to the original author(s) and the source, provide a link to the Creative Commons licence, and indicate if changes were made. The images or other third party material in this article are included in the article's Creative Commons licence, unless indicated otherwise in a credit line to the material. If material is not included in the article's Creative Commons licence and your intended use is not permitted by statutory regulation or exceeds the permitted use, you will need to obtain permission directly from the copyright holder. To view a copy of this licence, visit http://creativecommons.org/licenses/by/4.0/.

\section{References}

Alexander AL, Lee JE, Lazar M, Field AS (2007) Diffusion tensor imaging of the brain. Neurotherapeutics 4(3):316-329. https:// doi.org/10.1016/j.nurt.2007.05.011

Alexander DC, Dyrby TB, Nilsson M, Zhang H (2017) Imaging brain microstructure with diffusion MRI: practicality and applications. NMR Biomed 32(4):e3841. https://doi.org/10.1002/nbm.3841

Augoff K, Hryniewicz-Jankowska A, Tabola R (2015) Lactate dehydrogenase 5: an old friend and a new hope in the war on cancer. Cancer Lett 358(1):1-7. https://doi.org/10.1016/j.canlet.2014.12.035

Bailey HE (2020) Acute necrotizing encephalopathy associated with influenza A. Neurodiagn J 60(1):41-49. https://doi.org/10.1080/ 21646821.2020 .1725864

Beaud V, Crottaz-Herbette S, Dunet V et al (2020) Pattern of cognitive deficits in severe COVID-19. J Neurol Neurosurg Psychiatry. https://doi.org/10.1136/jnnp-2020-325173

Benameur K, Agarwal A, Auld SC et al (2020) Encephalopathy and encephalitis associated with cerebrospinal fluid cytokine alterations and coronavirus disease, Atlanta, Georgia, USA. Emerg Infect Dis 26(9):2016-2021. https://doi.org/10.3201/eid2609. 202122

Benjamini Y, Hochberg Y (1995) Controlling the false discovery rate: a practical and powerful approach to multiple testing. J R Stat Soc Series B Stat Methodol 57(1):289-300. http://www.jstor.org/ stable/2346101

Butowt R, Meunier N, Bryche B et al (2021) The olfactory nerve is not a likely route to brain infection in COVID-19: a critical review of data from humans and animal models. Acta Neuropathol 141(6):809-822. https://doi.org/10.1007/s00401-021-02314-2
Cannac O, Martinez-Almoyna L, Hraiech S (2020) Critical illnessassociated cerebral microbleeds in COVID-19 acute respiratory distress syndrome. Neurology 95(11):498-499. https://doi.org/10. 1212/WNL.0000000000010537

Clas P, Groeschel S, Wilke M (2012) A semi-automatic algorithm for determining the demyelination load in metachromatic leukodystrophy. Acad Radiol 19(1):26-34. https://doi.org/10.1016/j.acra. 2011.09.008

Eagen DE, Gonzales MM, Tarumi T et al (2012) Elevated serum C-reactive protein relates to increased cerebral myoinositol levels in middle-aged adults. Cardiovasc Psychiatry Neurol 2012:120540. https://doi.org/10.1155/2012/120540

Fotuhi M, Mian A, Meysami S, Raji CA (2020) Neurobiology of COVID-19. J Alzheimers Dis 76(1):3-19. https://doi.org/10.3233/ JAD-200581

Goldberg E, Podell K, Sodickson DK et al (2020) The brain after COVID-19: Compensatory neurogenesis or persistent neuroinflammation? EClinicalMedicine 31:100684. https://doi.org/10. 1016/j.eclinm.2020.100684

Helms J, Kremer S, Merdji H et al (2020a) Neurologic features in severe SARS-CoV-2 infection. N Engl J Med 382(23):2268-2270. https://doi.org/10.1056/NEJMc2008597

Helms J, Kremer S, Merdji H et al (2020b) Delirium and encephalopathy in severe COVID-19 patients: a cohort analysis of ICU patients. Crit Care 24:491. https://doi.org/10.1186/ s13054-020-03200-1

Iadecola C, Anrather J, Kamel H (2020) Effects of COVID-19 on the nervous system. Cell 183(1):16-27.e1. https://doi.org/10.1016/j. cell.2020.08.028

Kas A, Soret M, Pyatigoskaya $\mathrm{N}$ et al (2021) The cerebral network of COVID-19-related encephalopathy: a longitudinal voxel-based 18F-FDG-PET study. Eur J Nucl Med Mol Imaging 48(8):25432557. https://doi.org/10.1007/s00259-020-05178-y

Koll K, Willinger S, Urlesberger K, Pirker W (2020) Acute amnestic syndrome with hippocampal lesion due to influenza B-associated encephalopathy. Wien Klin Wochenschr 132(17-18):542-544. https://doi.org/10.1007/s00508-020-01721-x

Lampe L, Zhang R, Beyer F et al (2018) Visceral obesity relates to deep white matter hyperintensities via inflammation. Ann Neurol 85(2):194-203. https://doi.org/10.1002/ana.25396

López-Elizalde R, Campero A, Sánchez-Delgadillo T et al (2018) Anatomy of the olfactory nerve: a comprehensive review with cadaveric dissection. Clin Anat 31(1):109-117. https://doi.org/ 10.1002/ca.23003

Lu Y, Li X, Geng D et al (2020) Cerebral micro-structural changes in COVID-19 patients-an MRI-based 3-month follow-up study. EClinicalMedicine 25:100484. https://doi.org/10.1016/j.eclinm. 2020.100484

Mitaki S, Nagai A, Oguro H, Yamaguchi S (2015) C-reactive protein levels are associated with cerebral small vessel-related lesions. Acta Neurol Scand 133(1):68-74. https://doi.org/10.1111/ane. 12440

Mori S, Oishi K, Jiang H et al (2008) Stereotaxic white matter atlas based on diffusion tensor imaging in an ICBM template. Neuroimage 40(2):570-582. https://doi.org/10.1016/j.neuroimage. 2007.12.035

Motojima Y, Nagura M, Asano Y et al (2016) Diagnostic and prognostic factors for acute encephalopathy. Pediatr Int 58(11):11881192. https://doi.org/10.1111/ped.12995

Mukerji SS, Solomon IH (2021) What can we learn from brain autopsies in COVID-19? Neurosci Lett 742:135528. https://doi.org/10. 1016/j.neulet.2020.135528

Najjar S, Najjar A, Chong DJ et al (2020) Central nervous system complications associated with SARS-CoV-2 infection: integrative concepts of pathophysiology and case reports. J Neuroinflamm 17(1):231. https://doi.org/10.1186/s12974-020-01896-0 
Newcombe VFJ, Spindler LRB, Das T et al (2020) Neuroanatomical substrates of generalized brain dysfunction in COVID19. Intensive Care Med 47:116-118. https://doi.org/10.1007/ s00134-020-06241-w

Nitchingham A, Kumar V, Shenkin S et al (2018) A systematic review of neuroimaging findings in delirium: predictors, correlates and consequences. Int J Geriatr Psychiatry 33:1458-1478. https://doi. org/10.1002/gps.4724

Novikov DS, Fieremans E, Jesperen SN, Kiselev VG (2018) Quantifying brain microstructure with diffusion MRI: theory and parameter estimation. NMR Biomed 32(4):e3998. https://doi.org/10.1002/ nbm.3998

O’Donovan A, Bahorik A, Sidney S et al (2021) Relationships of inflammation trajectories with white matter volume and integrity in midlife. Brain Behav Immun 91:81-88. https://doi.org/10. 1016/j.bbi.2020.09.006

Parsons N, Outsikas A, Parish A et al (2020) Modelling the anatomic distribution of neurologic events in patients with COVID-19: a systematic review of MRI findings. AJNR Am J Neuroradiol 42(7):1190-1195. https://doi.org/10.3174/ajnr.A7113

Penny W, Friston K, Ashburner J, Kiebel S, Nichols T (2006) Statistical Parametric Mapping: The Analysis of Functional Brain Images. Elsevier, Amsterdam

Rahmanzade R, Lu PJ, Barakovic M et al (2020) Myelin and axon pathology in multiple sclerosis assessed by myelin water and multi-shell diffusion imaging. Brain. https://doi.org/10.1093/ brain/awab088

Raman B, Cassar MP, Tunnicliffe EM et al (2021) Medium-term effects of SARS-CoV-2 infection on multiple vital organs, exercise capacity, cognition, quality of life and mental health, post-hospital discharge. EClinicalMedicine 31:100683. https://doi.org/10. 1016/j.eclinm.2020.100683

Taquet M, Geddes JR, Husain M et al (2021) 6-month neurological and psychiatric outcomes in 236379 survivors of COVID-19: a retrospective cohort study using electronic health records. Lancet Psychiatry 8(5):416-427. https://doi.org/10.1016/S2215-0366(21) 00084-5

Teuwen LA, Geldhof V, Pasut A, Carmeliet P (2020) COVID-19: the vasculature unleashed. Nat Rev Immunol 20(7):389-391. https:// doi.org/10.1038/s41577-020-0343-0

Tsuchiya K, Katase S, Yoshino A, Hachiya J (2000) MRI of influenza encephalopathy in children: value of diffusion-weighted imaging. J Comput Assist Tomogr 24(2):303-307. https://doi.org/10.1097/ 00004728-200003000-00023

Valvona CJ, Fillmore HL, Nunn PB, Pilkington GJ (2016) The regulation and function of lactate dehydrogenase a: therapeutic potential in brain tumor. Brain Pathol 26(1):3-17. https://doi.org/10.1111/ bpa.12299

Wilson JE, Mart MF, Cunningham C et al (2020) Delirium. Nat Rev Dis Primers 6:90. https://doi.org/10.1038/s41572-020-00223-4

Publisher's Note Springer Nature remains neutral with regard to jurisdictional claims in published maps and institutional affiliations. 